\title{
Pragmatic trial of multifaceted intervention (STROKE-CARD care) to reduce cardiovascular risk and improve quality-of- life after ischaemic stroke and transient ischaemic attack -study protocol
}

Thomas Toell ${ }^{1}$, Christian Boehme ${ }^{1}$, Lukas Mayer ${ }^{1}$, Stefan Krebs², Clemens Lang ${ }^{2}$, Karin Willeit ${ }^{1,3}$, Barbara Prantl ${ }^{1}$, Michael Knoflach', Gerhard Rumpold ${ }^{4}$, Gudrun Schoenherr ${ }^{1}$, Andrea Griesmacher ${ }^{5}$, Peter Willeit ${ }^{1}$, Julia Ferrari ${ }^{2}$, Wilfried Lang ${ }^{2,6}$, Stefan Kiechl ${ }^{1 *}$ and Johann Willeit ${ }^{* *}$ (D)

\begin{abstract}
Background: Patients with ischaemic stroke or transient ischaemic attack (TIA) are at high risk of future cardiovascular events. Despite compelling evidence about the efficacy of secondary prevention, a substantial gap exists between risk factor management in real life and that recommended by international guidelines. Moreover, stroke is a leading cause of disability and morbidity which partly emerges from post-stroke complications.

Methods/design: We designed a block-randomised (2:1 ratio) open pragmatic trial [NCT02156778] with blinded outcome assessment comparing STROKE-CARD to usual post-stroke-patient care. STROKE-CARD is a multifaceted post-stroke disease management program with the objective of reducing recurrent cardiovascular events and improving quality of life in ischaemic stroke and TIA-patients. It combines intensified multi-domain secondary prevention, systematic detection and treatment of post-stroke complications, and patient self-empowerment. Enrolment of 2160 patients with acute ischaemic stroke or TIA (ABCD2-Score $\geq 3$ ) is planned at two study centres in Austria. The co-primary efficacy endpoints are (i) the composite of major recurrent cardiovascular events (nonfatal stroke, nonfatal myocardial infarction, and vascular death) occurring within 12 months after the index event and (ii) one-year health-related quality-of-life measured with the European Quality of Life-5 Dimensions (EQ-5D-3 L) questionaire. Secondary endpoints include allcause mortality, functional outcome, and target-level achievement in risk factor management.

Discussion: This trial will provide evidence on whether the pragmatic post-stroke intervention program STROKE-CARD can help prevent cardiovascular events and improve quality-of-life within the setting of a high-quality acute stroke care system. In case of success, STROKE-CARD may be implemented in daily clinical routine and serve as a model for other disease management initiatives.
\end{abstract}

Trial registration: ClinicalTrials.gov: NCT02156778. (June 5, 2014, retrospectively registered).

Keywords: Stroke, Transient ischemic attack, Secondary prevention, Disease management

\footnotetext{
* Correspondence: stefan.kiechl@i-med.ac.at; johann.willeit@i-med.ac.at

${ }^{1}$ Department of Neurology, Medical University of Innsbruck, Anichstraße 35,

A-6020 Innsbruck, Austria

Full list of author information is available at the end of the article
}

(c) The Author(s). 2018 Open Access This article is distributed under the terms of the Creative Commons Attribution 4.0 International License (http://creativecommons.org/licenses/by/4.0/), which permits unrestricted use, distribution, and reproduction in any medium, provided you give appropriate credit to the original author(s) and the source, provide a link to the Creative Commons license, and indicate if changes were made. The Creative Commons Public Domain Dedication waiver (http://creativecommons.org/publicdomain/zero/1.0/) applies to the data made available in this article, unless otherwise stated. 


\section{Background}

Stroke is the second leading cause of death and one of the leading contributors to disability worldwide $[1,2]$. While the age-standardised incidence of stroke is decreasing in high-income countries due to improved health care services and primary prevention, the absolute number of stroke patients is still on the rise, mainly based on continuous population aging and growth [3]. Stroke survivors represent a population-segment particularly vulnerable to further cerebro- and cardiovascular events. Apart from persistent deficits, potentially avoidable medium- and long-term post-stroke complications are significant contributors to functional impairment and an appealing target for concerted interventions.

The risk of stroke recurrence is high at up to $10 \%$ within one year and more than $25 \%$ within 5 years [4]. Recurrent strokes account for about one fifth of all strokes in state-wide registries [5], have a worse clinical outcome, have a higher fatality rate, and cause higher healthcare costs than first-ever strokes [6].

More than $90 \%$ of the global stroke burden is attributable to modifiable risk factors, including behavioural and metabolic factors $[7,8]$ and most patients with acute ischaemic stroke have one or more un- or insufficiently controlled risk factors - a condition recently termed "preventable stroke" [9]. Vice versa, current evidence-based secondary prevention strategies combining behavioural and pharmacological interventions were estimated to reduce the risk for recurrent vascular events by more than $80 \%$ [10]. In real life, however, prevention goals and target levels of risk factors are rarely achieved $[11,12]$. Furthermore, discontinuation of prescribed medications represents a major challenge in patient management, occurring in roughly one-third of ischaemic stroke patients within the first year of hospital discharge [13].

Multimodal and multi-disciplinary interventions within the framework of structured disease-management programs have been shown to improve quality of care and outcomes in patients with various chronic diseases other than stroke $[14,15]$. Also, digital health care interventions are a promising novel tool to improve individualised risk factor management [16].

To address the substantial gap between risk factor management in real life and that recommended by international guidelines, we designed the STROKE-CARD program, which is a multifaceted pragmatic post-stroke disease management program combining intensified multi-domain secondary prevention, detection and treatment of post-stroke complications, and patient self-empowerment. In a pragmatic trial, we compare STROKE-CARD to usual care in its ability to reduce rates of recurrent cardiovascular events and improve quality-of-life in ischaemic stroke and TIA-patients.

\section{Methods/design}

\section{Trial objectives}

In this trial, we test the hypothesis that the pragmatic disease-management program STROKE-CARD designed for ischaemic stroke and TIA patients is capable of (a) preventing recurrent cardiovascular events by optimised guideline-compliant secondary prevention and target level achievement and (b) ameliorating health-related quality of life (QoL) and patient-wellbeing by early detection and consequent treatment of post-stroke complications.

\section{Study design and centres}

STROKE-CARD is a randomised, controlled, open interventional phase III trial with blinded outcome assessment [ClinicalTrials.gov NCT02156778] conducted in Austria. It initially started as a single-centre study in January 2014 and was extended to a second centre in December 2014. It compares two standards of post-stroke patient care, both complying with the current state-of-the-art. The trial does not involve experimental interventions but compares usual with intensified care as specified below. Primary outcome events are adjudicated based on pre-specified diagnostic criteria blinded to treatment allocation.

The two trial centres are located at the Departments of Neurology at the Innsbruck University Hospital (IUH) and the Hospital St. John of God in Vienna. IUH serves as the comprehensive stroke unit for the entire federal state of Tyrol (catchment area $\approx 1 \mathrm{Mio}$ ) and as a primary stroke unit for the city of Innsbruck and 65 surrounding suburban communities with approximately 0.3 Mio inhabitants. Patients from this exclusive catchment area represent an unselected cohort of ischaemic stroke and TIA patients [5]. The Hospital St. John of God Vienna houses one of the four comprehensive stroke units in Vienna. The Viennese stroke network includes additional six primary stroke units and serves the city of Vienna with a catchment area of more than 1.8 Mio inhabitants.

\section{Study population}

The study population comprises consecutive patients with acute ischaemic stroke or TIA (ABCD2-Score $\geq 3$ ) [17] admitted to the two study centres. Patients are eligible for inclusion irrespective of whether the index event was a first or recurrent event. Ischaemic stroke is ascertained using the American Heart Association criteria based on clinical and imaging features [18].

All patients with ischaemic stroke or TIA admitted to the study centres are screened for potential inclusion in the STROKE-CARD trial during the first days of their hospital stay and in most instances included before discharge after signing an informed consent. Further details on the inclusion and exclusion criteria are provided in Table 1. 
Table 1 STROKE-CARD inclusion and exclusion criteria

\begin{tabular}{ll}
\hline Inclusion criteria & Exclusion criteria \\
\hline Patients with acute ischaemic stroke or TIA (ABCD2 Score $\geq 3$ points) & Patients living outside the catchment area \\
Age $\geq 18$ years & Malignancy or other severe disease with life-expectancy less than the \\
Signed informed consent & expected duration of the trial \\
& Drug addiction or severe alcohol abuse \\
& Patients with permanent severe disability and low perspectives for \\
& successful rehabilitation \\
& (mRS [19] $=5$ at discharge)
\end{tabular}

TIA transient ischaemic attack, mRS modified Rankin Scale [19]

\section{Randomisation and follow-up}

We block-randomise patients in a 2:1 ratio to receive the STROKE-CARD program or standard care on the basis of the exact date and time of the qualifying event, with the durations of the blocks ranging from 4 to 8 weeks. The target sample size is 2160 patients overall, 1440 patients in the STROKE-CARD group, and 720 in the control group (Fig. 1). We strive for inclusion of about 500 patients per year. Patients are followed-up for 12 months, but we also obtain consent from the patients to contact them later on and extend follow-up for future research projects. Patients who do not adhere to the extended standard care program, especially do not attend the 3-month assessment, are followed in the same way as adherent patients for primary and secondary outcome events.

\section{Intervention}

\section{Standard care}

In the control arm, patients are managed according to the usual stroke care protocol [5] of the study centres which includes: (a) detailed patient counselling and education about stroke pathophysiology, risk factor management, life style improvement and medication compliance by a stroke specialist (involving the next of kin and caregivers if appropriate); (b) provision of the complimentary book "After a stroke" [20] dealing with all aspects of stroke care; (c) individual in-hospital advice by a dietitian for patients with diabetes, severe dyslipidaemia, and obesity, and smoking cessation support for heavy smokers; (d) provision of standardised information materials (e.g. for oral anticoagulation therapy); and (e) provision at discharge of detailed medical reports for the general practitioner as well as the patient containing target levels for risk factor management. Post-discharge risk-factor-management is performed by the patient's general practitioner. Only selected high-risk patients are seen in the centres' outpatient clinics. A standardised 3-month telephone interview by a study nurse for assessment of functional outcome (mRS, Barthel Index, nursing allowance, life situation, support services, medication) is part of the country-wide quality program but has no interventional character [5]. As part of the STROKE-CARD protocol, all patients undergo a comprehensive standardised clinical visit 12 months after recruitment.

\section{Stroke-CARD}

In addition to the procedures listed above, the intervention-arm includes two additional interventions:

(a) a three-month outpatient appointment with standardised re-assessment of all risk factors and re-evaluation of stroke aetiology, screening for poststroke complications (e.g. spasticity, foot-drop, fatigue, pain, incontinence, communication problems, depression and anxiety, impaired cognition, seizures, fatigue, syncope, falls, fractures etc.), other health problems and residual deficits, estimation of the patient's demand for nursing services and the patient's adherence to drug prescriptions. All this information is used to optimise secondary prevention [21-23] with best possible achievement of target levels, to refine rehabilitation and treatment goals, and to manage post-stroke complications. Further details on the target levels are listed in Table 2. Patient assessment and counselling is done by a multidisciplinary team of physicians, nurses, physiotherapists, occupational and speech therapists. Treatment decisions are determined by experienced stroke neurologists. The overall visit takes about 3-4 h. The general practitioner receives a detailed medical report with precise instructions and treatment goals. Additional 6-month and 9-month visits are performed exceptionally (target $<5 \%$ of cases) at the discretion of the study team in case of medical need, complications requiring follow-up, or very poor risk factor control.

(b) Patients are offered access to an interactive password-protected web-based patient portal called "My Strokecard" [https://ches.tirol-kliniken.at/cms/ ]. If patients are unable to use a computer, caregivers are asked to assist with the usage of "My Strokecard". The patient-portal has been designed building on existing software for electronic patientreported outcome monitoring [24] and contains three components: (i) An adapted version of the 'post-stroke checklist' for ascertainment of poststroke complications is available for screening purposes at any time and pop up automatically three weeks before the three- and twelve-month 


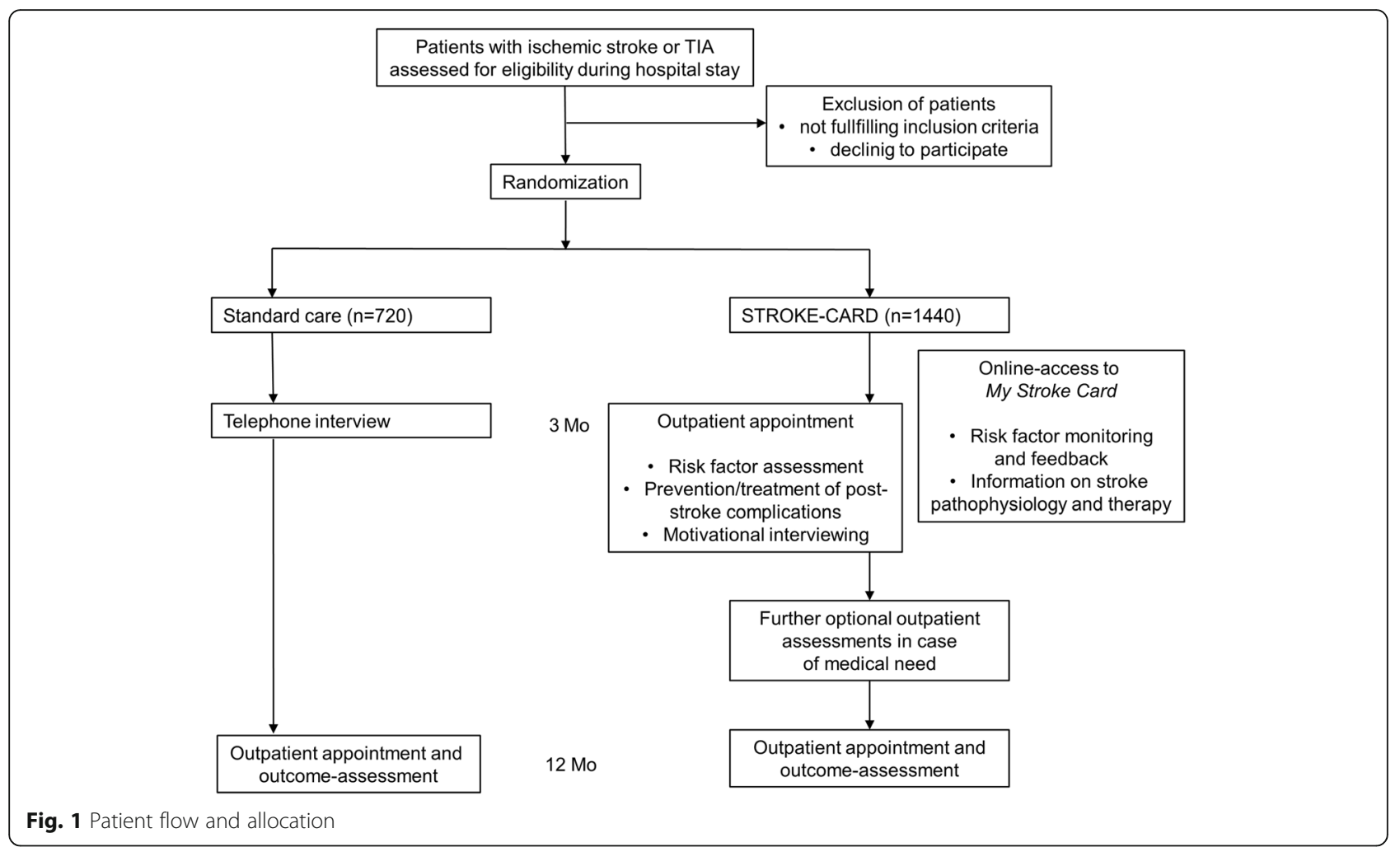

outpatient appointment, so that the readout of the questionnaires is already available before the medical visit. (ii) A self-administered internet-based tool for risk factor monitoring and patient selfempowerment (i.e. blood pressure, body weight, nicotine consumption, physical activity, LDL-C and HbA1c in case of diabetes) automatically generates feedback on target level achievement [tabular protocols and simple graphs visualising the current stage and time trends for the patient and GP, see Additional file 1]. (iii) Extensive information and educational materials on stroke pathophysiology, symptoms and risk factor management are provided.

During the in-hospital phase, clinical care does not differ between the two groups, with the exception of the training for the use of "My Stroke Card" in the extended care group before discharge.

There will be (i) a continuous monitoring of different benchmarks, including the duration of hospital stay and access to rehabilitation facilities to ensure that the two groups do not receive differential attention and to minimise surveillance biases, and (ii) a monitoring of trial progress by a data monitoring board.

\section{Outcome}

Primary outcomes (Co-primary endpoint)

- Incidence of major cardiovascular events defined as nonfatal stroke (ischaemic or haemorrhagic), nonfatal myocardial infarction (including acute coronary syndrome requiring emergency vascularisation), and vascular death (i.e. sudden cardiac death and death from acute myocardial infarction, ischaemic or haemorrhagic stroke, heart failure, cardiovascular procedures, pulmonary embolism, or peripheral artery disease) within one year of the index event.

- Health-related QoL assessed by the European Quality of Life-5 Dimensions (EQ-5D-3 L) overall health utility score one year after the index event.

\section{Secondary outcomes}

- Recurrent ischaemic or haemorrhagic stroke and TIA (defined as transient neurological deficit $<24 \mathrm{~h}$ and absence of DWI positive lesions on MRI) within one year of the index-event.

- All-cause mortality at 12 months.

- Favorable functional outcome (mRS $\leq 2$ and change in $\mathrm{mRS}$ ) at one year and at three months. 
Table 2 Target levels and interventions for risk factors at the 3-month risk factor assessment

\begin{tabular}{|c|c|c|}
\hline Condition & Target & Intervention to achieve the targets \\
\hline Hypertension & $\begin{array}{l}\mathrm{BP}<140 / 90 \mathrm{mmHg} \\
\mathrm{BP}<130 / 85 \mathrm{mmHg} \text { in patients with diabetes, } \\
\text { renal impairment or small-vessel disease }\end{array}$ & $\begin{array}{l}\text { Adjustment of anti-hypertensive medication } \\
\text { Information on supportive lifestyle changes } \\
\text { Written recommendations to and intensified } \\
\text { management by the GP }\end{array}$ \\
\hline Dyslipidaemia & $\begin{array}{l}\mathrm{LDL}-\mathrm{C}<100 \mathrm{mg} / \mathrm{dL} \\
\mathrm{LDL}-\mathrm{C}<70 \mathrm{mg} / \mathrm{dL} \text { in very high-risk patients }\end{array}$ & $\begin{array}{l}\text { Adjustment of statin dosage } \\
\text { Prescription of ezetimibe additionally to high-dose } \\
\text { statins (according to guidelines) } \\
\text { Involvement of a lipid-clinic and prescription of PCSK9 } \\
\text { inhibitors (according to guidelines) } \\
\text { Individualised recommendations by a dietitian }\end{array}$ \\
\hline Diabetes & $\mathrm{HbA}_{1 c}<7 \%$ & $\begin{array}{l}\text { Re-evaluation of the therapeutic regime by a diabetes- } \\
\text { specialist } \\
\text { Additional management by the GP, re-instruction in } \\
\text { correct administration of injectable pharmacological } \\
\text { agents } \\
\text { Individualised recommendations by a dietitian }\end{array}$ \\
\hline Smoking & Nicotine abstinence & $\begin{array}{l}\text { Motivational interviewing and counselling } \\
\text { Provision of informational material } \\
\text { Involvement of a psychiatric specialist for behavioural } \\
\text { or pharmacological therapy }\end{array}$ \\
\hline Physical inactivity & $\begin{array}{l}\text { Physical activity of moderate to vigorous } \\
\text { intensity with an average of } 40 \mathrm{~min} \text { at least } \\
3 \text { times per week }\end{array}$ & $\begin{array}{l}\text { Motivational interviewing } \\
\text { Provision of informational material }\end{array}$ \\
\hline Non-adherence to drug prescriptions & $\begin{array}{l}\text { Adherence to drug prescription (proportion } \\
\text { of days covered } \geq 90 \% \text { ) }\end{array}$ & $\begin{array}{l}\text { Motivational interviewing with information on indication } \\
\& \text { therapeutic effect of current medication } \\
\text { Simplification of drug-regimes } \\
\text { Home support by nurses and/or relatives }\end{array}$ \\
\hline $\begin{array}{l}\text { Poor "stroke-knowledge" of patients } \\
\& \text { family-members or caregivers }\end{array}$ & & $\begin{array}{l}\text { Information on stroke pathophysiology \& individual } \\
\text { stroke mechanism } \\
\text { Provision of a book and standardised information material }\end{array}$ \\
\hline $\begin{array}{l}\text { Post-stroke complications }{ }^{\mathrm{a}} \& \\
\text { poor functional outcome }\end{array}$ & Improvement of QoL & $\begin{array}{l}\text { Individualised treatment \& prevention of post-stroke } \\
\text { complications } \\
\text { Provision of further outpatient or inpatient rehabilitation } \\
\text { Re-assessment on nursing demands, social integration \& } \\
\text { outpatient care } \\
\text { Involvement of a social worker }\end{array}$ \\
\hline
\end{tabular}

$B P$ blood pressure, GP general practitioner, $L D L-C$ low-density lipoprotein cholesterol, $P C S K 9$ proprotein convertase subtilisin/kexin type $9, H b A_{1 c}$ glycated haemoglobin, QoL quality of life

${ }^{a}$ spasticity, pain, risk of falling, post stroke-depression (PSD), fatigue, anxiety, dysphagia, social deprivation, post-stroke dementia

- Individual components of the EQ-5D-3 L questionnaire, i.e. mobility, self-care, usual activities, pain and discomfort, anxiety and depression after one year.

- Achievement of predefined target levels in secondary prevention 12 months after the index event: $\mathrm{BP}<$ 140/90 $\mathrm{mmHg}$ [ $<130 / 85 \mathrm{mmHg}$ in selected patients], target $\mathrm{HbA}_{1 \mathrm{c}}$ in patients with diabetes [mainly $<7.0 \%$, less stringent targets in elderly], nicotine abstinence, LDL-C $<100 \mathrm{mg} / \mathrm{dL}$ [LDL-C $<70 \mathrm{mg} / \mathrm{dL}$ in high-risk patients] [23], physical activity with an average of $40 \mathrm{~min}$ at least 3 times per week, platelet inhibitor or anticoagulation according to ischaemic stroke aetiology [in case of oral anticoagulation with vitamin $\mathrm{K}$ antagonists: INR $2-3$, time in therapeutic range (TTR) > 70\%], statins except for patients with ischaemic strokes of non-atherosclerotic origin and no evidence of atherosclerosis (e.g. vessel dissection), medication adherence [proportion of days covered
$(\mathrm{PDC}) \geq 90 \%]$ [25]. The latter criterion focuses on statins, platelet inhibitors other than aspirin (which is typically purchased over the counter), antihypertensive medication, oral anti-diabetic drugs, and anticoagulation.

\section{Clinical assessments and study protocols}

The protocol uses validated and field-proven forms, scales and questionnaires. All the study team members are highly experienced and NIHSS- and mRS-certified.

The clinical baseline-assessment (entire study population) and the outpatient appointment (3-month intervention [intervention arm only] and 12-month outcome assessment [entire study population]) will be performed by trained health care professionals of the multidisciplinary study team composed of experienced stroke neurologists, PhD-students, physiotherapists, and stroke nurses. Data are collected prospectively according to pre-specified 


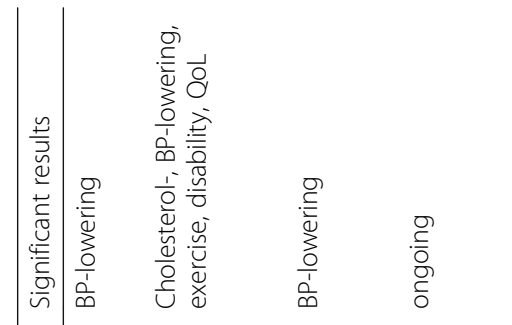

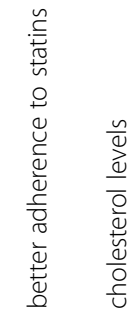

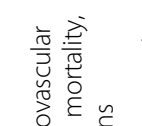







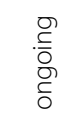

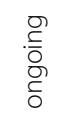

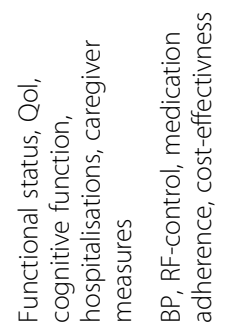

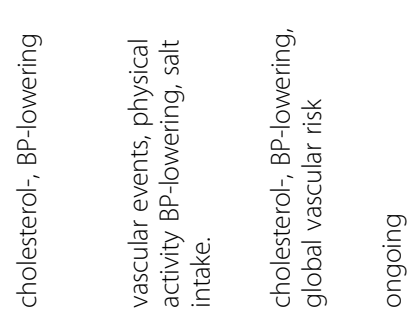

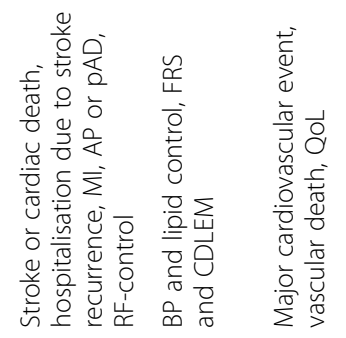

$\stackrel{\infty}{\infty} \simeq$

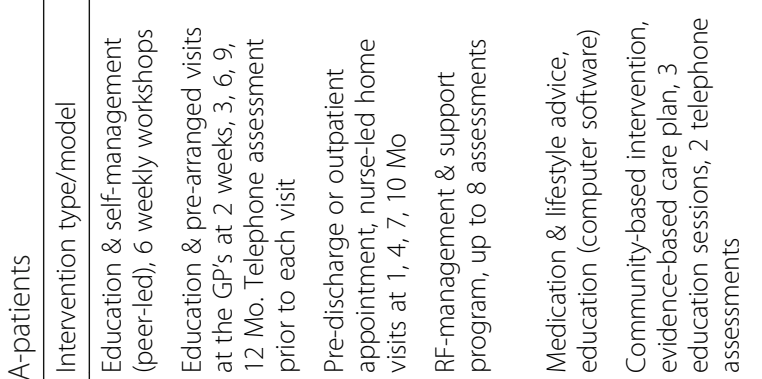

O)

ष्ष

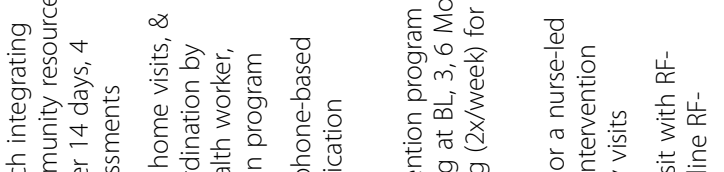



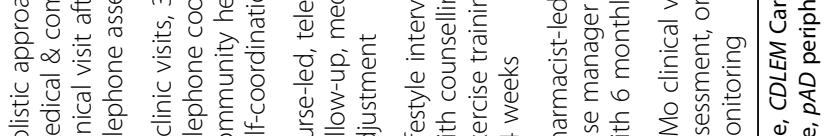

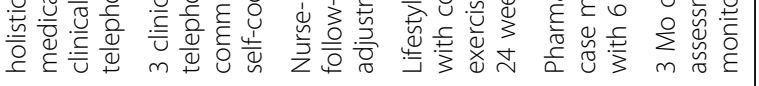

\& 范字

$\stackrel{\varangle}{z}$

$\infty \stackrel{\square}{i}$

$\stackrel{\$}{z}$

$\stackrel{\leftarrow}{\Sigma}$

in

$\infty \quad \hat{0} \quad \$$

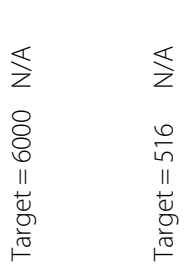

$\frac{1}{+\infty}$

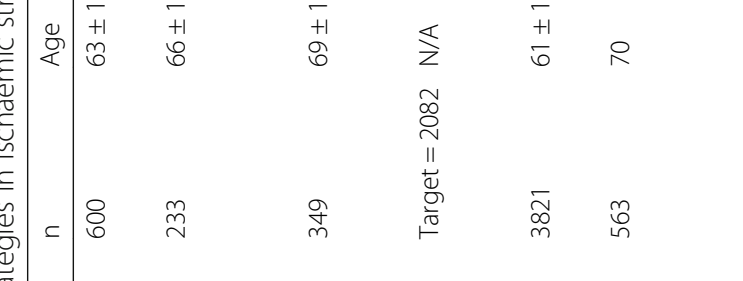

站

光

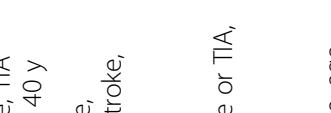

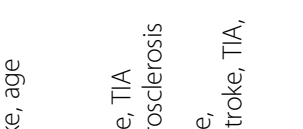

咅

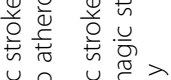

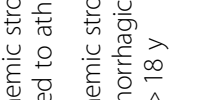

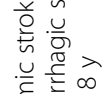

过荒宁

है है 항

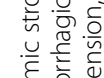

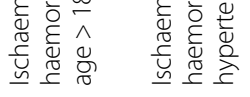

ฉे

ฉิ

ㅎํ స్

in

$\frac{1}{2+1}$

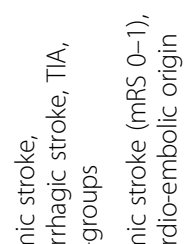



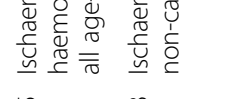

는

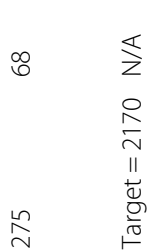


protocols and questionnaires with electronic data entry and storage and undergo subsequent quality checks and validation (e.g. by hospital records and feedback from the GP). All hospitals in the survey area dispose of electronic records, to which study investigators were granted access via protected data links according to the patients' informed consent.

The baseline assessment includes detailed documentation of the following components: ischaemic stroke aetiology [TOAST-criteria [26], Causative Classification of Stroke [27]], stroke severity [NIHSS [28] on admission and discharge], clinical syndrome [stroke/TIA symptoms, vessel territories], acute stroke therapy and recanalization procedures, pre-stroke disability and disability on admission [mRS], prior cardiovascular events and comorbidities, cardiovascular risk factors (hypertension, dyslipidaemia, smoking-status [current, former, never] and burden [pack-years, Fagerstroem questionnaire [29]], alcohol consumption [quantity, patterns, type of beverages], diabetes [ADA criteria] [30], atrial fibrillation, physical activity [Baecke Physical Activity Questionnaire] [31]), family history of cardiovascular disease, previous and current medication, cognitive function [Mini-Mental State Examination [32] and Montreal Cognitive Assessment [33]], depression [Beck Depression Inventar, anxiety [Hospital Anxiety and Depression Scale [34]], fatigue [Fatigue Severity Scale [35]], health related quality of life [European Quality of Life-5 Dimensions EQ5D-3 L [36]], anthropometric measures [body mass index, waist-to hip ratio], repeated blood pressure recordings [acute setting and before discharge], ankle-brachial index, atherosclerosis of the carotid arteries [intima-media thickness, plaque burden], [37] pulse wave velocity [Complior ${ }^{\circ}$ system], and routine laboratory measures [including lipid profile, renal function, liver function, thyroid function, cardiac markers, differential blood count, coagulation diagnostics, and inflammation markers].

The 3- and 12-month visits involve a neurological evaluation with documentation of clinical deficits and functional outcome [NIHSS, mRS, and Barthel Index [38]], routine laboratory examination and assessment of recurrent cardiovascular events, hospital stays and procedures [interventional and surgical], falls and fractures, all incident diseases and morbidities [including bleeding events], current medication, adherence to prescribed drugs, and risk factor control. Cognitive function, depression, anxiety, fatigue, and QoL all are re-evaluated [see baseline assessment]. Carotid ultrasound [see baseline protocol] is obligatorily repeated during the 12-month visit and, if clinically indicated, also after three months.

Blood and urine samples are drawn after an overnight fast and at least 12-h abstinence from smoking [during the hospital stay and 3 and 12 months thereafter], immediately processed, used for routine testing, and stored in a biobank [plasma, serum, whole blood, and urine]. Samples are stored at $-80{ }^{\circ} \mathrm{C}$ in compliance with the OECD guidelines and Austrian Bioethics Commission recommendations [39].

\section{Sample size}

Sample size calculations for this trial were based on the primary composite cardiovascular disease endpoint. Assuming a 1-year cumulative risk of $15 \%$ in the usual care group, 2045 subjects are required to detect a 5\% absolute risk reduction $(\alpha=0.05)$ with a $90 \%$ power.

Expecting an attrition rate of $15 \%$ (7.5\% dropout rate [withdrawal of consent] and a loss-to-follow-up of 7.5\%), we aimed to include a total of 2400 patients. Between January 2014 and June 2017, 1866 patients were recruited, with an attrition rate substantially lower than expected $(<5.0 \%$ as opposed to the anticipated $15 \%)$. Accordingly, the data monitoring board recommended revision of the recruitment goal from 2400 to 2160 patients. A sample size of 2160 patients provides the trial with a $90 \%$ power to detect a difference of 0.03 points on the EQ-5D-3 L overall health utility score (co-primary efficacy endpoint) assuming a standard deviation of 0.2 .

\section{Statistical analyses}

The primary efficacy analysis is based on time from hospital discharge to first occurrence of primary composite end-point as defined above. It is an unadjusted survival analysis according to the intention-to-treat principle with use of the log-rank test. Hazard ratios and 95\% confidence intervals will be estimated using Cox models with the assumption of proportional hazards checked by means of Schoenfeld residuals. Prespecified sensitivity analyses include adjusted models and a per-protocol analysis. Consistency of intervention effects in four prespecified subgroups (men and women, age groups, type of index event, and patients with or without regular use of My StrokeCard) will be assessed by means of test for interaction. The continuous efficacy endpoint QoL will be analysed with linear regression models.

Owing to the fact that our study attempts to encourage patients to adhere to current evidence-based prevention guidelines and does not include experimental treatment concepts, we do not perform a formal safety analysis. We however analyze whether there are any differences in the occurrence of potential side effects, risks and consequences of intensified secondary prevention in both study arms including (a) major bleeding [40], (b), syncopes and fractures, (c) laboratory abnormalities (ALT, CK, GFR), and (d) muscle-related outcomes. No formal interim analysis will be performed. All reported $P$ values are two-sided. 


\section{Discussion}

The risk of stroke recurrence within one year after ischaemic stroke or TIA is high and can effectively be reduced by effective guideline-compliant secondary prevention. Vice versa, poor adherence to guidelines leads to preventable recurrent events, worse outcome and higher health care costs. Post-stroke complications are frequent, often remain unrecognised and contribute to stroke disability and poor QoL.

\section{The STROKE-CARD concept}

STROKE-CARD is the first comprehensive pragmatic post-stroke disease management in Austria and, to our knowledge, one of the largest interventions of its kind worldwide. STROKE-CARD is addressing a real-life-cohort of ischaemic stroke- and TIA-patients with the primary objectives of enhancing adherence to prevention guidelines and thereby lowering rates of recurrent vascular events and post-stroke complications, and improving functional outcome and QoL. Whereas disease management programs traditionally rely on expert opinion, our initiative strives for a rigorous scientific evaluation involving outcome and health economy analyses. It pursues the concept that optimal acute stroke care extends to a thorough three-month assessment and individualised counselling. We employ a multidisciplinary but lean and cheap intervention leveraging contemporary e-technology and encouraging patient self-empowerment with the prospect of a widespread implementation in case of success. Access to Internet and social media is now broadly available and STROKE-CARD offers web-based educational patientoperated tools for risk factor to record and manage risk factors combined with linkage of patient data to medical caregivers. The STROKE-CARD concept was presented as an abstract at the European Stroke Organisation Conference 2018 [41].

\section{Other post-stroke disease management programs}

Previous trials on multimodal secondary prevention strategies in ischaemic stroke or TIA-patients have shown variable improvements in risk factor control or medication adherence but most were not designed and powered to analyse potential effects recurrent cardiovascular disease and stroke events. An overview on trials focusing on multimodal secondary stroke prevention is provided in Table 3 . Limitations shared by these trials include the usually limited sample size (443 median, IQR 347.3) [42-50] and the common focus on TIA or minor stroke patients only $[48,49,51]$.

Only one large-scale study has been completed so far [45] and enrolled more than 3800 ischaemic stroke and TIA-patients in 47 hospitals in China. In spite of a better adherence to statin-medications no difference in the hard clinical end points after one year of follow-up could be demonstrated.

Two ongoing studies deserve special consideration: (i) The Comprehensive Post-Acute Stroke Service (COMPASS) study, [52] a cluster-randomised trial involving 40 hospitals in North Carolina, plans enrolment of 6000 ischaemic and haemorrhagic stroke patients and investigates potential effects of the COMPASS care model (telephone follow-up, one clinic visit within two weeks after discharge, and an individualised patient electronic care plan) on the patient-reported 90-day functional outcome (primary endpoint), hospital re-admission rates, and 90-day mortality. (ii) The Intensified Secondary Prevention intending a Reduction of Recurrent Events in TIA and Minor Stroke patients (INSPiRE-TMS) study, [51] from Germany intends to recruit 2082 patients with minor stroke or TIA, provides up to eight appointments in outpatient clinics and focuses on two-year recurrent vascular events or vascular death.

Type and intensity of intervention vary widely among the different trials. Several programs envisage nurse-led home visits or telephone-based assessments for risk factor control [44, 47, 50]. The ICARUSS-strategy [43] in Australia trusts on several pre-arranged GP visits whereas the STANDFIRM-trial $(n=500)$, [46] a community-based intervention, consists of educational sessions, multiple follow-up assessments, and as telephone interviews conducted by study nurses and general practices. A recent intervention [49] has achieved a substantial and sustained (six months) reduction in global vascular risk (Framingham Risk Score, Cardiovascular Disease Life Expectancy Model) by non-physician provider case management. Digital health interventions (e.g. telemedicine, web-based strategies, email and- mobile phone-reminders) improved risk factor control in vascular diseases other than stroke [16].

\section{Strengths and limitations}

Key and in part unique features of STROKE-CARD are its comprehensive focus on both recurrent vascular events (risk factor control and compliance) and post-stroke complications and QoL, its lean and easily applicable intervention, and the inclusion of moderate and severe ischaemic stroke patients (all except those with permanent severe disability).

The primary analysis may be viewed as conservative in two respects: [1] It relies on a short one-year follow-up whereas potential effects are expected to increase over time. Extension of follow-up is a decided goal and fund raising is in progress [2]. Our program is realised within the framework of a highly developed stroke care setting and bears the challenge of a strong comparator group with high-quality management of ischaemic stroke/TIA patients. Although findings of our trial are not easily extrapolated to less developed stroke systems, the potential 
benefit of the intervention is presumably even higher on the low-quality stroke care background.

Our program is focused on ischaemic stroke but may serve as a model for other diseases sensitive to post-discharge management.

In case of success, the next steps would be a refinement of STROKE-CARD components and identification of subgroups with a health benefit and cost effectiveness in order to end up with a practicable tool for broad clinical implementation in the whole of Tyrol, Austria and beyond.

\section{Additional file}

Additional file 1: Pragmatic Trial of Multifaceted Intervention (STROKE CARD care) to Reduce Cardiovascular Risk and Improve Quality-of-Life after Ischaemic Stroke and Transient Ischaemic Attack -Study protocol. Excerpt of the interactive website "My Strokecard". (DOCX 389 kb)

\section{Abbreviations}

ACS: Acute coronary syndrome; ADLs: Activities of daily living; ALT: Alanine aminotransferase; AP: Angina pectoris; BL: Baseline; BP: Blood pressure; CDLEM: Cardiovascular Disease Life Expectancy Model; CK: Creatine kinase; DWI: Diffusion weighted imaging; EQ-5D-3L: European Quality of Life-5 Dimensions; FRS: Framingham Risk Score; FU: Follow up; GFR: Glomerular filtration rate; GP: General practitioner; HbA1c: Glycated haemoglobin; IUH: Innsbruck University Hospital; LDL-C: low-density lipoprotein cholesterol; MI: Myocardial infarction; Mo: Month; MRI: Magnet resonance imaging; mRS: Modified Rankin Scale; N/A: Not available; NIHSS: National institute of health stroke scale; PAD: Peripheral artery disease; PCSK9: proprotein convertase subtilisin/kexin type 9; PDS: Post stroke-depression; QoL: Quality of life; RF: Risk factor; SBP: Systolic blood pressure; TIA: Transient ischaemic attack; TOAST: Trial of Org 10,172 in Acute Stroke Treatment

\section{Acknowledgements}

Not applicable.

\section{Funding}

The Medical University of Innsbruck served as the sponsor of this study and received financial support from the university hospital (Tirol Kliniken), Tyrolean Health Insurance Company (TGKK), the Tyrol Health Care Funds (TGF), and unrestricted research grants from Boehringer Ingelheim, Otto Bock and Sanofi Aventis. The study centre in Vienna additionally received a grant from Bayer Healthcare. The sponsor and partners had no influence on the study design and clinical decisions, and no role in data analysis, interpretation, and publication

\section{Availability of data and materials}

The datasets used and/or analysed during the current study are available from the corresponding author on reasonable request.

\section{Authors' contribution}

$\Pi$, JW, SK, WL, and MK designed the study protocol and gained ethical approval. TT, CB, LM, SK, CL, KW, BP, GR, GS, AG, JF are involved in patient recruitment or data acquisition. PW coordinates data management and analysis. TT and CB performed the systematic review. $\Pi \pi$ wrote the first version of the manuscript. All authors reviewed and edited the manuscript and approved the final version of the manuscript.

\section{Ethics approval and consent to participate}

The study protocol was approved by the local Ethics Committee of the Medical University of Innsbruck in October 2013 (recruitment start, January 2014) and the local Ethics Committee of the Hospital St. John of God Vienna in December 2014 (recruitment start, December 2014). All patients gave their written informed consent for participation in the trial and the use of biosamples and data for future research projects targeting etiology, complications, outcome, prognosis, and management of ischaemic stroke and TIA.

\section{Consent for publication}

Not applicable.

\section{Competing interests}

The author(s) declared no potential conflicts of interest with respect to the research, authorship, and/or publication of this article.

\section{Publisher's Note}

Springer Nature remains neutral with regard to jurisdictional claims in published maps and institutional affiliations.

\section{Author details}

'Department of Neurology, Medical University of Innsbruck, Anichstraße 35, A-6020 Innsbruck, Austria. ${ }^{2}$ Department of Neurology, Hospital St. John of God, Johannes von Gott Platz 1, A-1020 Vienna, Austria. ${ }^{3}$ Department of Neurology, Inselspital Bern, University Hospital, Freiburgstrasse, CH-3010 Bern, Switzerland. ${ }^{4}$ Department of Medical Psychology, Medical University of Innsbruck, Schöpfstraße 23a, A-6020 Innsbruck, Austria. ${ }^{5}$ Central Institute of Medical and Chemical Laboratory Diagnostics, University Hospital of Innsbruck, Anichstraße 35, A-6020 Innsbruck, Austria. ${ }^{6}$ Sigmund Freud Private University, Medical Faculty, Campus Prater Freudplatz 1, A-1020 Vienna, Austria.

Received: 22 June 2018 Accepted: 21 October 2018

Published online: 06 November 2018

\section{References}

1. Hay SI. Global, regional, and national incidence, prevalence, and years lived with disability for 328 diseases and injuries for 195 countries, 1990-2016: a systematic analysis for the global burden of disease study 2016. Lancet. 2017;390:1211-59.

2. Steel N. Global, regional, and national age-sex specific mortality for 264 causes of death, 1980-2016: a systematic analysis for the global burden of disease study 2016. Lancet. 2017:390(10100):1151-210.

3. Feigin VL, Forouzanfar MH, Krishnamurthi R, Mensah GA, Connor M, Bennett DA, et al. Global and regional burden of stroke during 1990-2010: findings from the global burden of disease study 2010. Lancet. 2014;383(9913): 245-55.

4. Mohan KM, Wolfe CDA, Rudd AG, Heuschmann PU, Kolominsky-Rabas PL, Grieve AP. Risk and cumulative risk of stroke recurrence: a systematic review and meta-analysis. Stroke. 2011:42(5):1489-94.

5. Willeit J, Geley T, Schöch J, Rinner H, Tür A, Kreuzer H, et al. Thrombolysis and clinical outcome in patients with stroke after implementation of the Tyrol stroke pathway: a retrospective observational study. Lancet Neurol. 2015:14(1):48-56.

6. Samsa GP, Bian J, Lipscomb J, Matchar DB. Epidemiology of recurrent cerebral infarction a Medicare claims-based comparison of first and recurrent strokes on 2-year survival and cost. Stroke. 1999;30(2):338-49.

7. Feigin VL, Roth GA, Naghavi M, Parmar P, Krishnamurthi R, Chugh $S$, et al. Global burden of stroke and risk factors in 188 countries, during 1990-2013: a systematic analysis for the Global Burden of Disease Study 2013. Lancet Neurol [Internet]. 2016 [cited 2016 Nov 16]; Available from: https://doi.org/ 10.1016/S1474-4422(16)30073-4.

8. O'Donnell MJ, Chin SL, Rangarajan S, Xavier D, Liu L, Zhang H, et al. Global and regional effects of potentially modifiable risk factors associated with acute stroke in 32 countries (INTERSTROKE): a case-control study. Lancet. 2016;388(10046):761-75

9. Fisher M, Moores L, Alsharif MN, Paganini-Hill A. Definition and implications of the preventable stroke. JAMA Neurol. 2016;73(2):186.

10. Hackam DG, Spence JD. Combining multiple approaches for the secondary prevention of vascular events after stroke: a quantitative modeling study. Stroke. 2007;38(6):1881-5.

11. Heuschmann PU, Kircher J, Nowe T, Dittrich R, Reiner Z, Cifkova R, et al. Control of main risk factors after ischaemic stroke across Europe: data from the stroke-specific module of the EUROASPIRE III survey. Eur J Prev Cardiol. 2015;22(10):1354-62

12. on behalf of the ASPIRE-S Study Group, Brewer L, Mellon L, Hall P, Dolan E, Horgan F, et al.. Secondary prevention after ischaemic stroke: the ASPIRE-S 
study. BMC Neurol [Internet]. 2015 Dec [cited 2016 Nov 16];15(1). from: https:/doi.org/10.1186/s12883-015-0466-2

13. Bushnell CD, Olson DM, Zhao X, Pan W, Zimmer LO, Goldstein LB, et al. Secondary preventive medication persistence and adherence 1 year after stroke. Neurology. 2011;77(12):1182-90.

14. Gonseth J, Guallar-Castillón P, Banegas JR, Rodríguez-Artalejo F. The effectiveness of disease management programmes in reducing hospital readmission in older patients with heart failure: a systematic review and meta-analysis of published reports. Eur Heart J. 2004;25(18):1570-95.

15. Riedl R, Robausch M, Berghold A. The Evaluation of the Effectiveness of Austrians Disease Management Program in Patients with Type 2 Diabetes Mellitus - A Population-Based Retrospective Cohort Study. Reboldi G. PLOS ONE. 2016;11(8):e0161429.

16. Widmer RJ, Collins NM, Collins CS, West CP, Lerman LO, Lerman A. Digital health interventions for the prevention of cardiovascular disease: a systematic review and meta-analysis. Mayo Clin Proc. 2015;90(4):469-80.

17. DeLange T, Frech $\mathrm{G}$, Kelen $\mathrm{G}$. Validation and refinement of scores to predict very early stroke risk after transient ischaemic attack. Lancet. 2007;369:283-92.

18. Sacco RL, Kasner SE, Broderick JP, Caplan LR, Connors JJ, Culebras A, et al. An updated definition of stroke for the 21st century: a statement for healthcare professionals from the American Heart Association/American Stroke Association. Stroke. 2013;44(7):2064-89.

19. Van Swieten JC, Koudstaal PJ, Visser MC, Schouten HJ, Van Gijn J. Interobserver agreement for the assessment of handicap in stroke patients. Stroke. 1988; 19(5):604-7.

20. Kiechl S, Lalouschek W, Lang W. NACH EINEM SCHLAGANFALL. 2nd ed. Holzhausen Verlag; 2014. 152 p.

21. Kernan WN, Ovbiagele B, Black HR, Bravata DM, Chimowitz MI, Ezekowitz $M D$, et al. Guidelines for the prevention of stroke in patients with stroke and transient ischemic attack. Stroke. 2014;45(7):2160-236.

22. Van Horn L, Carson JAS, Appel LJ, Burke LE, Economos C, Karmally W, et al.. Recommended Dietary Pattern to Achieve Adherence to the American Heart Association/American College of Cardiology (AHA/ACC) Guidelines: A Scientific Statement From the American Heart Association. Circulation. 2016; CIR.0000000000000462.

23. Catapano AL, Graham I, De Backer G, Wiklund O, Chapman MJ, Drexel H, et al. 2016 ESC/EAS guidelines for the Management of Dyslipidaemias. Eur Heart J. 2016:37(39):2999-3058.

24. Holzner B, Giesinger JM, Pinggera J, Zugal S, Schöpf F, Oberguggenberger AS, et al. The computer-based health evaluation software (CHES): a software for electronic patient-reported outcome monitoring. BMC Med Inform Decis Mak. 2012;12(1):126.

25. Choudhry NK, Shrank WH, Levin RL, Lee JL, Jan SA, Brookhart MA, et al. Measuring concurrent adherence to multiple related medications. Am J Manag Care. 2009;15(7):457-64.

26. Adams HP, Bendixen BH, Kappelle LJ, Biller J, Love BB, Gordon DL, et al. Classification of subtype of acute ischemic stroke. Definitions for use in a multicenter clinical trial. TOAST. Trial of org 10172 in acute stroke treatment. Stroke. 1993;24(1):35-41.

27. Ay $H$, Benner T, Murat Arsava E, Furie KL, Singhal AB, Jensen MB, et al. A computerized algorithm for etiologic classification of ischemic stroke: the causative classification of stroke system. Stroke. 2007;38(11):2979-84.

28. Lyden P, Brott T, Tilley B, Welch KM, Mascha EJ, Levine S, et al. Improved reliability of the NIH stroke scale using video training. NINDS TPA Stroke Study Group Stroke. 1994;25(11):2220-6.

29. Heatherton TF, Kozlowski LT, Frecker RC, K-O F. The Fagerström test for nicotine dependence: a revision of the Fagerstrom tolerance questionnaire. Br J Addict. 1991;86(9):1119-27.

30. American Diabetes Association. Diagnosis and Classification of Diabetes Mellitus. Diabetes Care. 2010;33(Supplement_1):S62-9.

31. Baecke JA, Burema J, Frijters JE. A short questionnaire for the measurement of habitual physical activity in epidemiological studies. Am J Clin Nutr. 1982; 36(5):936-42.

32. Folstein MF, Folstein SE, McHugh PR. "Mini-mental state": a practical method for grading the cognitive state of patients for the clinician. J Psychiatr Res. 1975;12(3):189-98.

33. Nasreddine ZS, Phillips NA, Bédirian V, Charbonneau S, Whitehead V, Collin I, et al. The Montreal cognitive assessment, MoCA: a brief screening tool for mild cognitive impairment. J Am Geriatr Soc. 2005;53(4):695-9.

34. Zigmond AS, Snaith RP. The hospital anxiety and depression scale. Acta Psychiatr Scand. 1983;67(6):361-70.
35. Krupp LB, LaRocca NG, Muir-Nash J, Steinberg AD. The fatigue severity scale: application to patients with multiple sclerosis and systemic lupus erythematosus. Arch Neurol. 1989;46(10):1121-3.

36. Brooks R, Group E. EuroQol: the current state of play. Health Policy. 1996; 37(1):53-72.

37. Willeit K, Pechlaner R, Egger G, Weger S, Oberhollenzer M, Willeit J, et al. Carotid atherosclerosis and incident atrial fibrillation. Arterioscler Thromb Vasc Biol. 2013;33(11):2660.

38. Collin C, Wade DT, Davies S, Horne V. The Barthel ADL index: a reliability study. Int Disabil Stud. 1988;10(2):61-3.

39. OECD Organization for Economic Cooperation and Development. Eur J Health Law. 2010;17(2):191-204.

40. Schulman S, Kearon C, The subcommittee on control of anticoagulation of the scientific and standardization committee of the international society on thrombosis and haemostasis. Definition of major bleeding in clinical investigations of antihemostatic medicinal products in non-surgical patients. J Thromb Haemost. 2005:3(4):692-4.

41. European Stroke Organisation Conference. Late Breaking Abstracts. Eur Stroke J. 2018;3(1_suppl):587-620.

42. Kronish IM, Goldfinger JZ, Negron R, Fei K, Tuhrim S, Arniella G, et al. The effect of peer education on stroke prevention: the prevent recurrence of all Inner-City strokes through education (PRAISE) randomized controlled trial. Stroke J Cereb Circ. 2014;45(11):3330-6.

43. Joubert J, Reid C, Barton D, Cumming T, McLean A, Joubert L, et al. Integrated care improves risk-factor modification after stroke: initial results of the integrated Care for the Reduction of secondary stroke model. $J$ Neurol Neurosurg Amp Psychiatry. 2009;80(3):279.

44. Hornnes N, Larsen $\mathrm{K}$, Boysen $\mathrm{G}$. Blood pressure 1 year after stroke: the need to optimize secondary prevention. J Stroke Cerebrovasc Dis. 2011;20(1):16-23.

45. Peng B, Ni J, Anderson CS, Zhu Y, Wang Y, Pu C, et al. Implementation of a structured guideline-based program for the secondary prevention of ischemic stroke in China. Stroke. 2014;45(2):515-9.

46. Olaiya MT, Cadilhac DA, Kim J, Nelson MR, Srikanth VK, Gerraty RP, et al. Community-based intervention to improve Cardiometabolic targets in patients with stroke. Stroke. 2017;48(9):2504.

47. Irewall A-L, Ögren J, Bergström L, Laurell K, Söderström L, Mooe T. NurseLed, Telephone-Based, Secondary Preventive Follow-Up after Stroke or Transient Ischemic Attack Improves Blood Pressure and LDL Cholesterol: Results from the First 12 Months of the Randomized, Controlled NAILED Stroke Risk Factor Trial. Pizzi C, editor. PLOS ONE. 2015;10(10):e0139997.

48. Kono Y, Yamada S, Yamaguchi J, Hagiwara Y, Iritani N, Ishida S, et al. Secondary prevention of new vascular events with lifestyle intervention in patients with Noncardioembolic mild ischemic stroke: a single-center randomized controlled trial. Cerebrovasc Dis. 2013;36(2):88-97.

49. McAlister FA, Grover S, Padwal RS, Youngson E, Fradette M, Thompson A, et al. Case management reduces global vascular risk after stroke: secondary results from the the preventing recurrent vascular events and neurological worsening through intensive organized case-management randomized controlled trial. Am Heart J. 2014;168(6):924-30.

50. Rochette A, Korner-Bitensky N, Bishop D, Teasell R, White CL, Bravo G, et al. The YOU CALL-WE CALL randomized clinical trial: impact of a multimodal support intervention after a mild stroke. Circ Cardiovasc Qual Outcomes. 2013;6(6):674-9.

51. Leistner S, Michelson G, Laumeier I, Ahmadi M, Smyth M, Nieweler G, et al. Intensified secondary prevention intending a reduction of recurrent events in TIA and minor stroke patients (INSPiRE-TMS): a protocol for a randomised controlled trial. BMC Neurol. 2013;13(1):11.

52. Duncan PW, Bushnell CD, Rosamond WD, Jones Berkeley SB, Gesell SB, D'Agostino RB, et al. The comprehensive post-acute stroke services (COMPASS) study: design and methods for a cluster-randomized pragmatic trial. BMC Neurol. 2017;17:133.

53. Towfighi A, Cheng EM, Ayala-Rivera M, McCreath H, Sanossian N, Dutta T, et al. Randomized controlled trial of a coordinated care intervention to improve risk factor control after stroke or transient ischemic attack in the safety net: secondary stroke prevention by uniting community and chronic care model teams early to end disparities (SUCCEED). BMC Neurol. 2017;17:24. 